\title{
Lack of Association between TLR4 Genetic Polymorphisms and Diabetic Nephropathy in a Chinese Population
}

\author{
Danfeng Peng, Jie Wang, Jiemin Pan, Rong Zhang, Shanshan Tang, Feng Jiang, Miao Chen, \\ Jing Yan, Xue Sun, Tao Wang, Shiyun Wang, Yuqian Bao, and Weiping Jia
}

\author{
Shanghai Diabetes Institute, Shanghai Key Laboratory of Diabetes Mellitus, Shanghai Clinical Center for Diabetes, \\ Shanghai Key Clinic Center for Metabolic Diseases, Department of Endocrinology and Metabolism, Shanghai Jiao Tong University \\ Affiliated Sixth People's Hospital, Shanghai 200233, China
}

Correspondence should be addressed to Weiping Jia; wpjia@sjtu.edu.cn

Received 26 January 2014; Accepted 28 February 2014; Published 23 March 2014

Academic Editor: Cheng $\mathrm{Hu}$

Copyright ( 2014 Danfeng Peng et al. This is an open access article distributed under the Creative Commons Attribution License, which permits unrestricted use, distribution, and reproduction in any medium, provided the original work is properly cited.

Objective. Toll-like receptor 4 (TLR4) plays a central role in innate immunity. Activation of innate immune response and subsequent chronic low-grade inflammation are thought to be involved in the pathogenesis of diabetic nephropathy. In this study, we aimed to investigate whether TLR4 variants are associated with diabetic nephropathy in the Chinese population. Methods. Seven tagging single nucleotide polymorphisms (SNPs) of TLR4 based on HapMap Chinese data were genotyped in 1,455 Chinese type 2 diabetic patients. Of these patients, 622 were diagnosed with diabetic nephropathy and 833 were patients with diabetes for over 5 years but without diabetic nephropathy. Results. None of the SNPs and haplotypes showed significant association to diabetic nephropathy in our study. No association between the SNPs and quantitative traits was observed either. Conclusion. We concluded that common variants within TLR4 genes were not associated with diabetic nephropathy in the Chinese type 2 diabetes patients.

\section{Introduction}

Type 2 diabetes has become an epidemic all around the world, resulted in large loss in economy, and threatened the human health. Diabetic microvascular complications are the major causes of morbidity and early mortality in diabetes $[1,2]$. As one of the most important long-term complications of diabetes, diabetic nephropathy is the leading cause of chronic kidney failure and end-stage renal disease [3], and patients with diabetic nephropathy have an increased risk of cardiovascular mortality $[4,5]$. At present, it is widely accepted that diabetic nephropathy is a heterogeneous disorder caused by the interaction between environmental factors and genetic factors. Diabetes duration and glycemic control are the strongest environmental risk factors [6], and family history of kidney disease appears to be the strongest risk factor for initiation of diabetic nephropathy $[7,8]$. Recently, several studies have suggested that the innate immunity changes may be associated with type 2 diabetes and diabetic complications
[9-11]. Thus, genes encoding the innate immune system components might be good candidates for studying diabetic nephropathy.

Toll-like receptors (TLRs) are the family of type I transmembrane receptors involved in innate immunity and pathogen recognition [12]. While toll-like receptor 4 (TLR4) is predominantly expressed on dendritic cells and macrophages and plays an important role in the activation of the innate immune response and subsequent proinflammatory reactions, it not only recognizes the lipopolysaccharide (LPS) of Gram-negative bacteria, but also interacts with some endogenous ligands, such as heat-shock proteins, fibronectin, oxidized low-density lipoprotein cholesterol, Fetuin-A, and high-mobility group box 1 (HMGB1) [12-17]. Several studies have indicated that there may be a link between TLR4 pathway and diabetic nephropathy [18-20]. Therefore, in this study, we aim to investigate whether TLR4 genetic polymorphisms are associated with diabetic nephropathy and its related quantitative traits in the Chinese population. 
TABLE 1: Clinical characteristics of the study samples.

\begin{tabular}{lcc}
\hline & Cases & Controls \\
\hline Samples $(n)$ & 621 & 832 \\
Male/female $(n)$ & $348 / 274$ & $352 / 480$ \\
Age (years) & $62.42 \pm 12.67$ & $63.31 \pm 10.56$ \\
BMI (years) & $24.95 \pm 3.86$ & $24.00 \pm 3.35$ \\
Age at diagnosis of diabetes (years) & $53.56 \pm 12.27$ & $42.38 \pm 10.22$ \\
Duration of diabetes (years) & $9.00(3.00,14.00)$ & $10.00(7.00,14.00)$ \\
Hemoglobin A $(\%)$ & $9.34 \pm 2.29$ & $8.68 \pm 2.10$ \\
Systolic blood pressure (mmHg) & $140.04 \pm 19.27$ & $134.52 \pm 18.27$ \\
Diastolic blood pressure (mmHg) & $82.53 \pm 9.90$ & $79.57 \pm 9.05$ \\
AERs (mg/24 H) & $93.09(47.47,296.27)$ & $9.23(6.40,14.58)$ \\
eGFR & $109.81(81.62,136.87)$ & $121.57(103.40,144.84)$ \\
\hline
\end{tabular}

Data are $n$, mean \pm SD, or median (interquartile range). BMI: body mass index. AERs: albumin excretion rates. eGFR: estimated glomerular filtration rate. eGFR ${ }^{*}$ was calculated by using a formula developed by the Modification of Diet in Renal Disease study group with adjustment for Chinese ethnicity.

\section{Methods}

2.1. Participants. This study involved 1,455 patients with type 2 diabetes recruited from the Shanghai Diabetes Institute Inpatient Database of Shanghai Jiao Tong University Affiliated Sixth People's Hospital. All participants were unrelated type 2 diabetic patients meeting the 1999 WHO criteria. Of these patients, 622 were diagnosed with diabetic nephropathy and 833 were patients with diabetes for over 5 years but without diabetic nephropathy, considered as cases and controls for diabetic nephropathy, respectively. This study was approved by the institutional review board of Shanghai Jiao Tong University Affiliated Sixth People's Hospital, with written informed consent obtained from each participant.

2.2. Clinical Measurement. The $24 \mathrm{~h}$ albumin excretion rates (AERs) and estimated glomerular filtration rate (eGFR) were applied to asses nephropathy. AERs were measured in 3 consecutive days, and the mean value was recorded for each patient. Patients with AER $<30 \mathrm{mg} / 24 \mathrm{~h}, 30 \mathrm{mg} / 24 \mathrm{~h} \leq \mathrm{AER}$ $<300 \mathrm{mg} / 24 \mathrm{~h}$, or AER $\geq 300 \mathrm{mg} / 24 \mathrm{~h}$ were classified as having normoalbuminuria, microalbuminuria, or proteinuria, respectively. Patients having microalbuminuria or proteinuria were diagnosed with diabetic nephropathy. eGFR was calculated using a formula developed by the Modification of Diet in Renal Disease study group with adjustment for Chinese ethnicity: $186 \times$ (serum creatinine in $\mathrm{mmol} / \mathrm{L} \times$ $0.011)^{-1.154} \times(\text { age in years })^{-0.203} \times(0.742$ if female $) \times(1.233$ if Chinese) [21]. Glycemic control was evaluated by measuring glycated HbAlc levels. Data of blood pressures and lipid profiles were also collected for each participant.

\subsection{Single Nucleotide Polymorphisms (SNPs) Selection, Geno-} typing, and Quality Control. We selected tagging SNPs according to HapMap phase III (release 28) Han Chinese database with a threshold of $r^{2}>0.8$ by using Haploview (v 4.2). The seven tagging SNPs selected could cover $100 \%$ of common SNPs (14 out of 14 SNPs in the HapMap Chinese Han samples) with a minor allele frequency (MAF) $>0.05$. All genotyping was done using the primer extension of multiplex products with detecting by matrix-assisted laser desorption ionization time of flight mass spectroscopy using a MassARRAYCompact Analyzer (Sequenom, San Diego, CA, USA). The genotyping data underwent a series of quality control checks as described previously [22] and cleared data were used in further statistical analysis. Overall, 2 individuals were excluded from the sample call rate checks and all the seven SNPs passed the SNP call rate check with an average call rate of $98 \%$.

2.4. Statistical Analysis. The Hardy-Weinberg equilibrium test was performed before the association analysis (a twotailed $P$ value $<0.05$ was considered statistically significant). The allelic frequencies between the patients with or without diabetic nephropathy were compared by $\chi^{2}$ test, and odds ratios with $95 \%$ confidence intervals (CIs) were presented. Linear regression was applied to test the effect of genotype on quantitative traits with adjustment of confounding factors under an additive model. Skewly distributed quantitative traits (eGFR and AER) were logarithmically transformed $(\log 10)$ to approximate normality before linear regression analysis. All these analyses were performed using SAS 9.3 (SAS institute, Cary, NC, USA) unless specified otherwise. A two-tailed $P$ value $<0.05$ was considered statistically significant.

\section{Results}

All the seven SNPs were in accordance with Hardy-Weinberg equilibrium. The clinical characteristics of the samples passed genotype quality control were shown in Table 1 . The linkage disequilibrium pattern of these SNPs was shown in Figure 1. Two haplotype blocks were constructed in this region.

The single SNP association analysis showed that no SNP was significantly associated with diabetic nephropathy in our samples. The minimum $P$ value was 0.417 for rs7044464 (Table 2). Then we compared the frequencies of haplotypes between type 2 diabetic patients with or without diabetic nephropathy and observed that there was no nominal difference between two groups either (Table 3). Further, we 


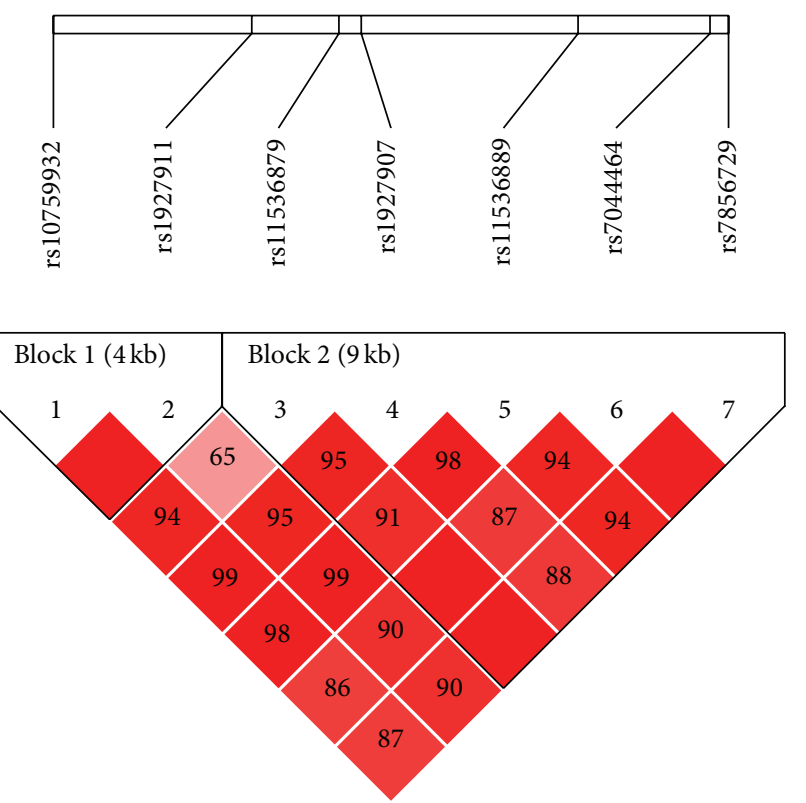

(a)

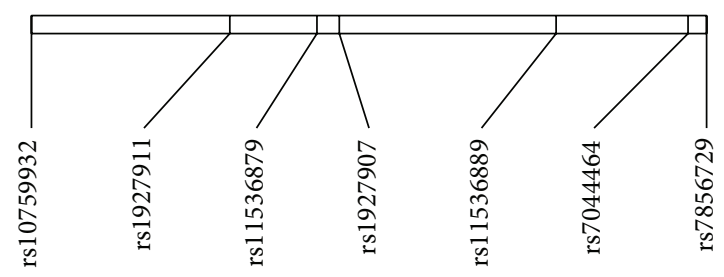

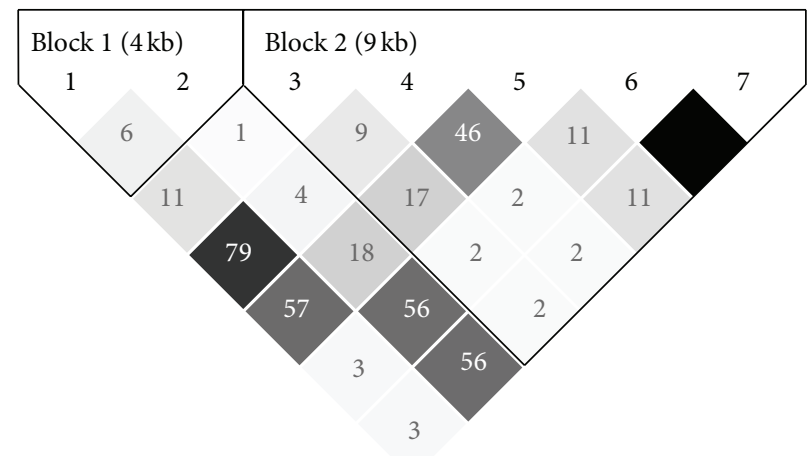

(b)

FIGURE 1: Linkage disequilibrium maps for SNPs genotyped in TLR4 region. (a) Shades of red demonstrate the strength of the pairwise linkage disequilibrium based on $\mathrm{D}^{\prime}$ and numbers represent the value of $\mathrm{D}^{\prime}$ expressed as a percentage. (b) Shades of grey show the strength of the pairwise linkage disequilibrium based on $r^{2}$ and numbers indicate the value of $r^{2}$ expressed as a percentage.

analyzed the effect of these SNPs on nephropathy related quantitative traits. With adjustment of age, sex, body mass index, duration of diabetes, HbAlC, diastolic pressure, and systolic pressure, we found that no SNP was nominally associated with AER and eGFR. The minimum $P$ value was 0.087 for the association between rs10759932 and AER (Table 4).

\section{Discussion}

It has been shown that TLR4 and its signal pathway participated in the pathogenesis of diabetes and diabetic nephropathy. Dasu et al. [23] reported that TLR4 expression and its ligand, signaling, and functional activation were increased in recently diagnosed type 2 diabetes subjects and contributed to the proinflammatory state. Furthermore, knockout of tlr4 attenuated the proinflammatory state of diabetes in animal models [24]. Exposure of isolated islets of Langerhans to LPS reduced insulin gene expression; insulin secretion was inhibited as well. However, those effects were not observed in islets from TLR4-deficient mice [25]. In vitro, TLR4 expression and activity were increased under hyperglycemia in mesangial cells and could contribute to the progression of diabetic nephropathy [18]. Liu et al. [20] observed that renal TLR4 expression was significantly higher in diabetic nephropathy in animal model, as well as kidney/body weight ratio, serum creatinine, CRP, and TNF- $\alpha$ level. TLR4 has also been proved to accelerate the progression of diabetic nephropathy induced by hyperlipidemia [26]. The research of Lin et al. [17] also showed that TLR4 pathway promoted tubular inflammation in diabetic nephropathy. And their further study investigated that TLR4 antagonist CRX-526 could reduce albuminuria and blood urea nitrogen without altering blood glucose and systolic blood pressure in diabetic mice, thus protecting diabetic mice from advanced nephropathy [19]. Above all, it is convincible that TLR4 and its signal pathway play an important role in diabetic nephropathy.

The data of TLR4 genetic polymorphisms and diabetic complications was limited. It was reported in a German population that Asp299Gly and Thr399Ile genotypes of the TLR4 were associated with diabetic neuropathy in type 2 diabetes, but not with diabetic nephropathy [27]. Asp299Gly was associated with early onset of diabetic retinopathy in the type 2 patients, reported in a Poland population [28]. However, these two polymorphisms were not detected in the Chinese population [29-32]. In the present study, we aimed to investigate the association between TLR4 polymorphisms and diabetic nephropathy. However, we failed to find any evidence of association between SNPs from this locus and traits related to diabetic nephropathy in our samples. One possible explanation might be that the statistical power of our samples was not enough to detect the effects of this locus in the Chinese population. With our samples, we had over $75 \%$ power to detect an effect SNP $(\mathrm{OR}=1.4)$ with minor allele frequency of 0.1 at a 0.05 level. We could not exclude the possibility that associated SNPs with lower minor allele frequency or lower effect in the Chinese existed. Secondly, although the tagging SNPs we selected covered all the common SNPs of $T L R 4$, it is still possible that rare variants of TLR4 associated with diabetic nephropathy existed. Thirdly, in our study, the diagnosis of diabetic nephropathy was not determined by the histological analysis of tissue samples obtained from renal 


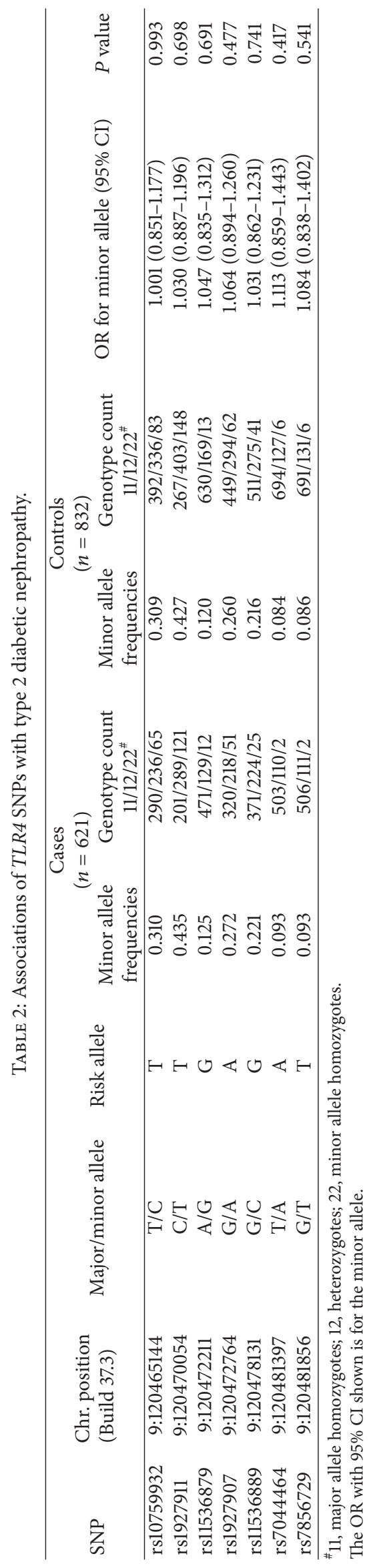


TABLE 3: Associations of two haplotypes in TLR4 region with diabetic nephropathy.

\begin{tabular}{lrr}
\hline Haplotype & \multicolumn{2}{c}{ Haplotype frequencies } \\
Controls & Cases & 0.570 \\
Block 1 (rs10759932-rs1927911) & & 0.309 \\
TA & 0.566 & 0.827 \\
CA & 0.309 & 0.973 \\
TG & 0.125 & 0.705 \\
Block 2 (rs11536879-rs1927907-rs11536889-rs7044464-rs7856729) & & 0.361 \\
GGCTG & 0.342 & 0.253 \\
GATTG & 0.269 & 0.288 \\
CGCTG & 0.215 & 0.341 \\
GGTAT & 0.088 & 0.5205 \\
GGTTG & 0.071 & 0.080 \\
\hline
\end{tabular}

TABLE 4: Associations of TLR4 SNPs with clinical features related to diabetic nephropathy in type 2 diabetic patients without nephropathy.

\begin{tabular}{|c|c|c|c|c|c|c|}
\hline \multirow{2}{*}{ SNP } & \multicolumn{3}{|c|}{ AERs } & \multicolumn{3}{|c|}{ eGFR } \\
\hline & Beta & SE & $P$ value ${ }^{\#}$ & Beta & SE & $P$ value ${ }^{\#}$ \\
\hline rs10759932 & -0.022 & 0.013 & 0.087 & 0.003 & 0.006 & 0.683 \\
\hline rs1927911 & -0.011 & 0.012 & 0.354 & 0.002 & 0.006 & 0.781 \\
\hline rs11536879 & 0.019 & 0.019 & 0.312 & -0.001 & 0.009 & 0.894 \\
\hline rs1927907 & -0.019 & 0.013 & 0.165 & 0.001 & 0.006 & 0.839 \\
\hline rs11536889 & 0.000 & 0.015 & 0.974 & -0.003 & 0.007 & 0.712 \\
\hline rs7044464 & 0.022 & 0.022 & 0.314 & 0.002 & 0.011 & 0.865 \\
\hline rs7856729 & 0.024 & 0.022 & 0.271 & 0.006 & 0.011 & 0.581 \\
\hline
\end{tabular}

The additive model was used in the analysis.

$P$ value ${ }^{\#}$ was adjusted for age, sex, BMI, diabetes duration, HbA1C, and blood pressure.

biopsies, which is the golden standard [33]. So we cannot exclude the possibility that patients diagnosed with diabetic nephropathy also may include nondiabetic renal disease and a superimposed nondiabetic condition on underlying diabetic nephropathy. However, we excluded patients with history of renal diseases in the enrollment of study subjects, and the blood pressures of patients with or without diabetic nephropathy were similar, and thus the influence of the diagnostics of diabetic nephropathy on our study was limited.

\section{Conclusions}

In conclusion, although many functional researches have implied that TLR4 played an important role in diabetic nephropathy, our study suggested that common variants within TLR4 gene were not associated with diabetic nephropathy in the Chinese type 2 diabetes patients. However, due to the limitation of the current study, the effects of SNPs from this locus on diabetic nephropathy needed to be tested in further studies with larger samples with accurate diagnosis for diabetic nephropathy.

\section{Conflict of Interests}

The authors declare that there is no conflict of interests regarding the publication of this paper.

\section{Authors' Contribution}

Danfeng Peng, Jie Wang, and Jiemin Pan contribute equally to this work.

\section{Acknowledgment}

This work was supported by Grants from the National 863 program (2012AA02A509), 973 Program (2011CB504001), NSFC (81200582 and 81300691), the Drug Innovation Program of the National Science and Technology Project (2011ZX09307-001-02), and Shanghai Health and Family Planning Commission (2013ZYJB1001). The authors appreciate all the participants of this research. The authors gratefully acknowledge the skillful technical support of all nursing and medical staff at Shanghai Clinical Center for Diabetes.

\section{References}

[1] Z. He and G. L. King, "Microvascular complications of diabetes," Endocrinology and Metabolism Clinics of North America, vol. 33, no. 1, pp. 215-238, 2004.

[2] A. Girach, D. Manner, and M. Porta, "Diabetic microvascular complications: can patients at risk be identified? A review," International Journal of Clinical Practice, vol. 60, no. 11, pp. 14711483, 2006. 
[3] E. Ritz, "Nephropathy in type 2 diabetes," Journal of Internal Medicine, vol. 245, no. 2, pp. 111-126, 1999.

[4] H.-H. Parving, J. T. Harrington, J. Lewis et al., "Diabetic nephropathy: prevention and treatment," Kidney International, vol. 60, no. 5, pp. 2041-2055, 2001.

[5] G. Remuzzi, A. Schieppati, and P. Ruggenenti, "Nephropathy in patients with type 2 diabetes," New England Journal of Medicine, vol. 346, no. 15, pp. 1145-1151, 2002.

[6] I. M. Stratton, A. I. Adler, H. A. W. Neil et al., "Association of glycaemia with macrovascular and microvascular complications of type 2 diabetes (UKPDS 35): prospective observational study," British Medical Journal, vol. 321, no. 7258, pp. 405-412, 2000.

[7] E. R. Seaquist, F. C. Goetz, S. Rich, and J. Barbosa, "Familial clustering of diabetic kidney disease. Evidence for genetic susceptibility to diabetic nephropathy," New England Journal of Medicine, vol. 320, no. 18, pp. 1161-1165, 1989.

[8] B. I. Freedman, N. V. Volkova, S. G. Satko et al., "Populationbased screening for family history of end-stage renal disease among incident dialysis patients," American Journal of Nephrology, vol. 25, no. 6, pp. 529-535, 2005.

[9] A. D. Pradhan, J. E. Manson, N. Rifai, J. E. Buring, and P. M. Ridker, "C-reactive protein, interleukin 6, and risk of developing type 2 diabetes mellitus," Journal of the American Medical Association, vol. 286, no. 3, pp. 327-334, 2001.

[10] H. Kolb and T. Mandrup-Poulsen, "An immune origin of type 2 diabetes?" Diabetologia, vol. 48, no. 6, pp. 1038-1050, 2005.

[11] M. K. Arora and U. K. Singh, "Molecular mechanisms in the pathogenesis of diabetic nephropathy: an update," Vascular Pharmacology, vol. 58, no. 4, pp. 259-271, 2013.

[12] T. Kawai and S. Akira, "The role of pattern-recognition receptors in innate immunity: update on toll-like receptors," Nature Immunology, vol. 11, no. 5, pp. 373-384, 2010.

[13] K. Hoshino, O. Takeuchi, T. Kawai et al., "Cutting edge: tolllike receptor 4 (TLR4)-deficient mice are hyporesponsive to lipopolysaccharide evidence for TLR4 as the Lps gene product," Journal of Immunology, vol. 162, no. 7, pp. 3749-3752, 1999.

[14] Y. Okamura, M. Watari, E. S. Jerud et al., "The extra domain a of fibronectin activates toll-like receptor 4," Journal of Biological Chemistry, vol. 276, no. 13, pp. 10229-10233, 2001.

[15] Y. Kumagai and S. Akira, "Identification and functions of pattern-recognition receptors," Journal of Allergy and Clinical Immunology, vol. 125, no. 5, pp. 985-992, 2010.

[16] D. Pal, S. Dasgupta, R. Kundu et al., "Fetuin-A acts as an endogenous ligand of TLR4 to promote lipid-induced insulin resistance," Nature Medicine, vol. 18, no. 8, pp. 1279-1285, 2012.

[17] M. Lin, W. H. Yiu, H. J. Wu et al., “Toll-like receptor 4 promotes tubular inflammation in diabetic nephropathy," Journal of the American Society of Nephrology, vol. 23, no. 1, pp. 86-102, 2012.

[18] H. Kaur, A. Chien, and I. Jialal, "Hyperglycemia induces Toll like receptor 4 expression and activity in mouse mesangial cells: relevance to diabetic nephropathy," American Journal of Physiology-Renal Physiology, vol. 303, no. 8, pp. F1145-F1150, 2012.

[19] M. Lin, W. H. Yiu, R. X. Li et al., "The TLR4 antagonist CRX526 protects against advanced diabetic nephropathy," Kidney International, vol. 83, no. 5, pp. 887-900, 2013.

[20] S. Y. Liu, X. Z. Nie, W. Y. Zhou et al., "Expression and effect of TLR4 in rats with diabetic nephropathy," Asian Pacific Journal of Tropical Medicine, vol. 6, no. 8, pp. 635-639, 2013.

[21] Y. C. Ma, L. Zuo, J. H. Chen et al., "Modified glomerular filtration rate estimating equation for Chinese patients with chronic kidney disease," Journal of the American Society of Nephrology, vol. 17, no. 10, pp. 2937-2944, 2006.

[22] C. Hu, C. Wang, R. Zhang et al., "Association of genetic variants of NOS1AP with type 2 diabetes in a Chinese population," Diabetologia, vol. 53, no. 2, pp. 290-298, 2010.

[23] M. R. Dasu, S. Devaraj, S. Park, and I. Jialal, "Increased TollLike Receptor (TLR) activation and TLR ligands in recently diagnosed type 2 diabetic subjects," Diabetes Care, vol. 33, no. 4, pp. 861-868, 2010.

[24] S. Devaraj, P. Tobias, and I. Jialal, "Knockout of toll-like receptor-4 attenuates the pro-inflammatory state of diabetes," Cytokine, vol. 55, no. 3, pp. 441-445, 2011.

[25] J. Amyot, M. Semache, M. Ferdaoussi, G. Fontés, and V. Poitout, "Lipopolysaccharides impair insulin gene expression in isolated islets of langerhans via toll-like receptor- 4 and nf- $\kappa$ b signalling," PLoS ONE, vol. 7, no. 4, Article ID e36200, 2012.

[26] T. Kuwabara, K. Mori, M. Mukoyama et al., "Exacerbation of diabetic nephropathy by hyperlipidaemia is mediated by Tolllike receptor 4 in mice," Diabetologia, vol. 55, no. 8, pp. 22562266, 2012.

[27] G. Rudofsky Jr., P. Reismann, S. Witte et al., "Asp299Gly and Thr399Ile genotypes of the TLR4 gene are associated with a reduced prevalence of diabetic neuropathy in patients with type 2 diabetes," Diabetes Care, vol. 27, no. 1, pp. 179-183, 2004.

[28] M. Buraczynska, I. Baranowicz-Gaszczyk, J. Tarach, and A. Ksiazek, "Toll-like receptor 4 gene polymorphism and early onset of diabetic retinopathy in patients with type 2 diabetes," Human Immunology, vol. 70, no. 2, pp. 121-124, 2009.

[29] X. D. Fu, X. Q. Sun, H. Y. Wang et al., "Genetic polymorphisms of the TLR4 gene and their association with susceptibility to type 2 diabetes mellitus in the Chinese population," Genetics and Molecular Research, vol. 12, no. 3, pp. 3813-3820, 2013.

[30] H. Cai, J. Cai, and G. Tao, "Association of toll-like receptor 4 polymorphisms with type 2 diabetes mellitus," APMIS, vol. 121, no. 7, pp. 605-611, 2013.

[31] Z. S. Jiang, S. X. Wang, H. X. Jia et al., "Association of tolllike receptor 4 polymorphisms with type 2 diabetes mellitus," Inflammation, vol. 36, no. 1, pp. 251-257, 2013.

[32] F. Liu, W. Lu, Q. Qian et al., "Frequency of TLR 2, 4, and 9 gene polymorphisms in Chinese population and their susceptibility to type 2 diabetes and coronary artery disease," Journal of Biomedicine and Biotechnology, vol. 2012, Article ID 373945, 7 pages, 2012.

[33] M. L. Gonzalez Suarez, D. B. Thomas, L. Barisoni et al., "Diabetic nephropathy: is it time yet for routine kidney biopsy?" World Journal of Diabetes, vol. 4, no. 6, pp. 245-255, 2013. 

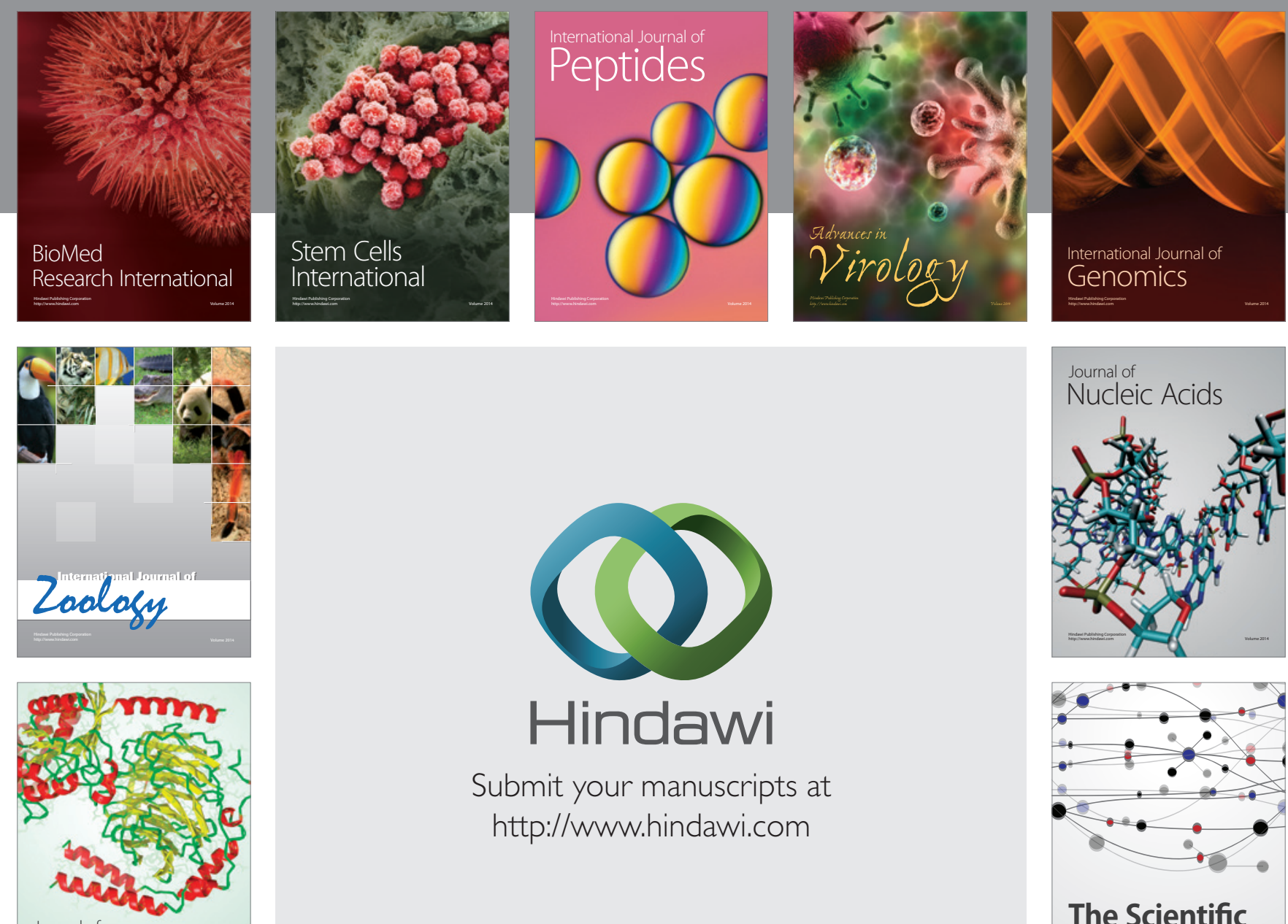

Submit your manuscripts at

http://www.hindawi.com

Journal of
Signal Transduction
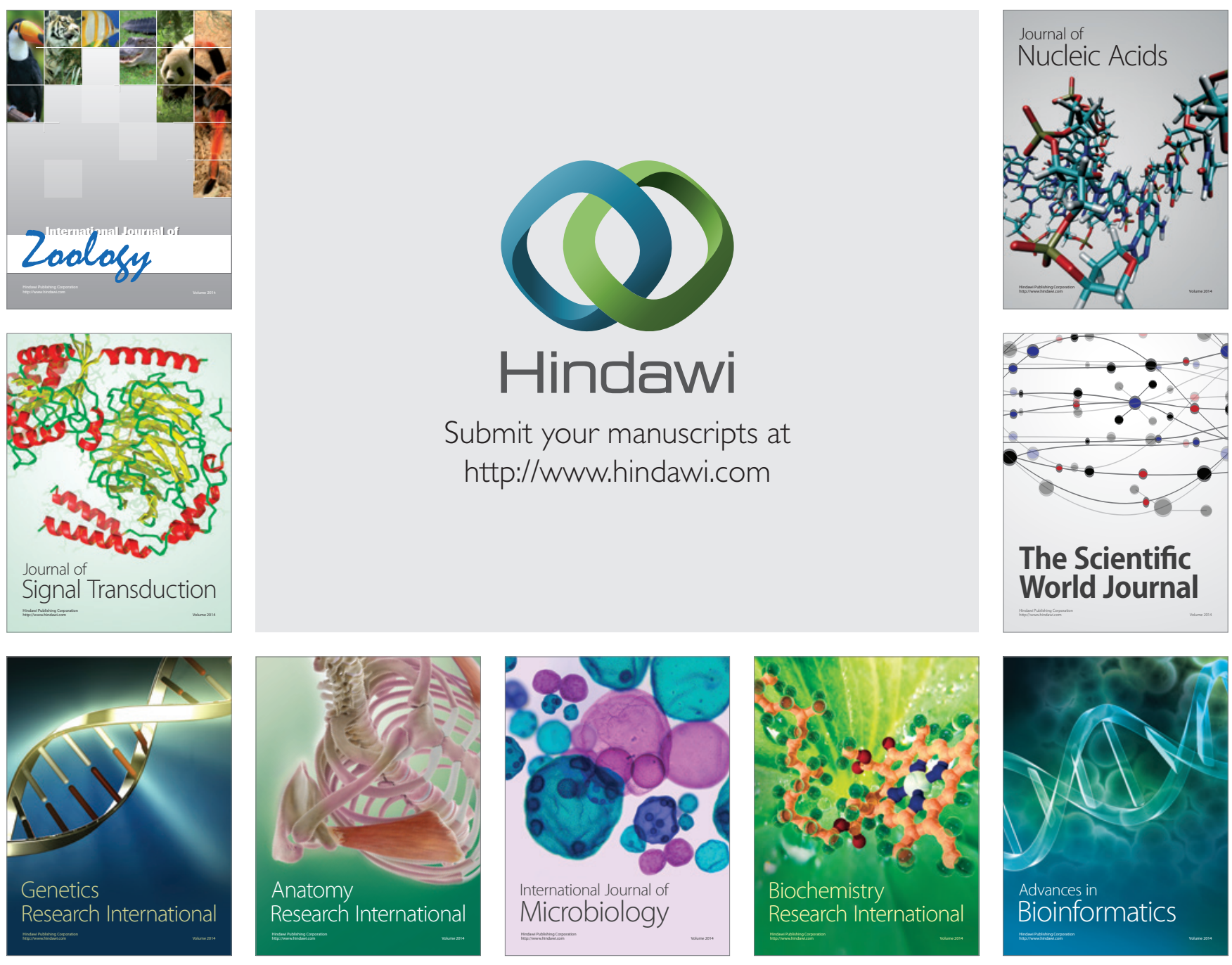

The Scientific World Journal
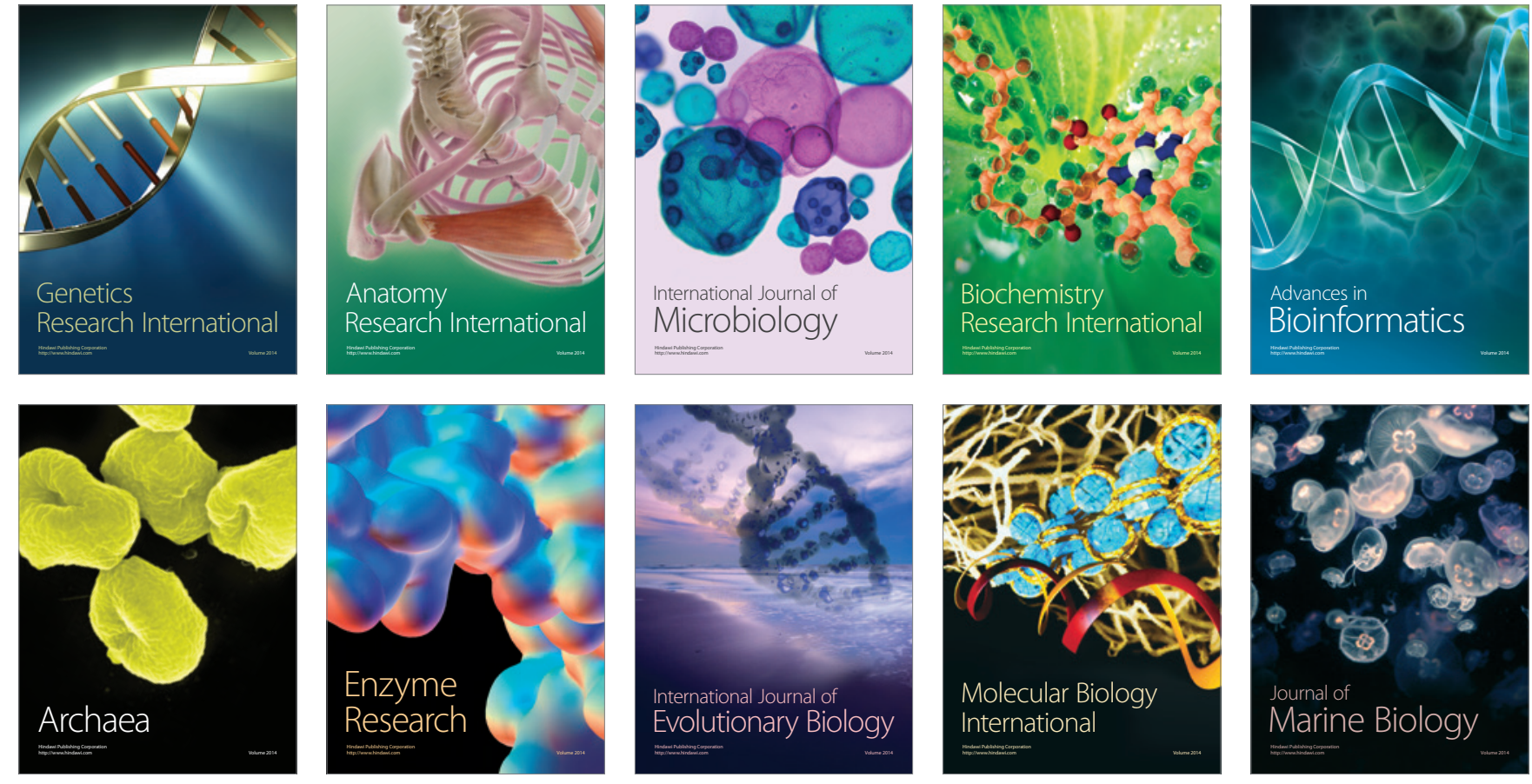